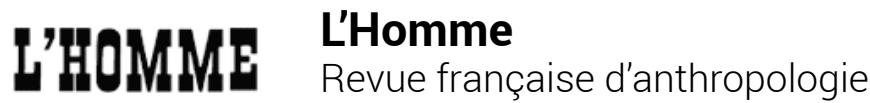

195-196 | 2010

Auto-biographie, Ethno-biographie

\section{Le moment néoréaliste de l'anthropologie démartinienne}

The Neorealistic Moment in De Martino's Anthropology

\section{Giordana Charuty}

\section{(2) OpenEdition}

Journals

\section{Édition électronique}

URL : http://journals.openedition.org/lhomme/22514

DOI : 10.4000/lhomme.22514

ISSN : 1953-8103

Éditeur

Éditions de l'EHESS

\section{Édition imprimée}

Date de publication : 10 novembre 2010

Pagination : 247-281

ISSN : 0439-4216

\section{Référence électronique}

Giordana Charuty, «Le moment néoréaliste de l'anthropologie démartinienne », L'Homme [En ligne], 195-196 | 2010, mis en ligne le 04 novembre 2012, consulté le 30 avril 2019. URL : http:// journals.openedition.org/lhomme/22514 ; DOI : 10.4000/lhomme.22514 


\title{
Le moment néoréaliste de l'anthropologie démartinienne
}

\author{
Giordana Charuty
}

\begin{abstract}
66 L' L HISTOIRE DE l'anthropologie comme problème anthropologique ":
cette formule, empruntée à Alfred I. Hallowell, définissait pour George W. Stocking les objectifs d'un programme de recherche novateur visant à explorer, de l'intérieur, chaque moment d'émergence ou de reconfiguration, au sein du monde occidental, d'un savoir systématique de «l'unité de l'homme dans la diversité » (Stocking 1983). Tout en partageant des principes adoptés, au même moment, par l'histoire sociale des sciences et des techniques, les études qui ont, alors, interrogé l'émergence de la notion moderne de terrain ethnographique, comme marqueur distinctif de la discipline, ont privilégié le récit biographique fragmentaire pour faire apparaître, dans le prisme d'une vie, l'inquiétude interprétative suscitée par des expériences inédites de dépaysement. C'est, par exemple, la longue lettre-journal écrite, entre 1883 et 1884, par le jeune Franz Boas à sa fiancée qui documente, mieux que d'autres écrits professionnels, les dimensions imaginaires de sa première rencontre avec les populations inuit de l'île de Baffin (Cole 1983). Ou bien, ce sont les conditions de vie domestique chez les Zunis qui éclairent certaines modalités du passage de Franz Cushing à un nouveau style ethnographique (Hinsley 1983).
\end{abstract}

Comme dans les autres sciences, les vies de fondateurs d'un moment et d'un style - Tylor, Frazer, Durkheim, Malinowski, Mauss, Leenhardt ont, par ailleurs, fait l'objet d'amples restitutions, fondées sur l'entrecroisement de diverses catégories d'archives. Elles furent d'abord le fait de chercheurs anglo-saxons et nord-américains qui n'ont pas hésité à considérer des auteurs vivants, par exemple Mary Douglas, tandis que des demandes éditoriales ou des intérêts plus éloignés de l'histoire disciplinaire ont conduit, en France, des universitaires et des journalistes à composer les 
vies de Michel Leiris, Germaine Tillion ou Claude Lévi-Strauss ${ }^{1}$. Les plus anciennes de ces biographies érudites conservent le modèle, longtemps en usage dans les études littéraires, de « la vie et l'œuvre » pour mettre l'accent sur l'exposé chronologique de la production intellectuelle d'un savant. Les plus récentes s'efforcent de "penser l'œuvre dans son contexte" pour éviter la surconsécration héroïque toujours susceptible de faire retour dans une entreprise de narration totalisante sans, cependant, aller au-delà d'une caractérisation extérieure des espaces savants pensés comme largement dominés par la rationalité pratique et par une conception volontariste de l'activité scientifique (Fournier 1994, 2007). En revanche, la nouvelle attention portée par les anthropologues aux archives de leurs prédécesseurs les conduit à emprunter, à leur tour, la voix biographique pour repenser les continuités et les ruptures qui sous-tendent les moments de reconfiguration des savoirs et de redéfinition des élites culturelles ${ }^{2}$. S'agissant de figures fondatrices comme Paul Rivet, ces études décrivent des itinéraires au sein d'espaces sociaux fragmentés où la construction, scientifique et institutionnelle, d'une nouvelle discipline compose avec des expériences existentielles, des convictions éthiques, des affinités personnelles, des engagements politiques (Laurière 2008). Ou bien, s'agissant de retracer la genèse française d'un domaine régional, elles considèrent avec la même attention des figures oubliées et de grands administrateurs ethnographes ${ }^{3}$.

1. Michael W. Young (2004) qualifie de "littéraire» - et non d'intellectuelle - sa biographie de Malinowski. Tout en cherchant dans l'éducation catholique de Mary Douglas l'expérience vécue de la prégnance des institutions sociales, la biographie que lui consacre l'anthropologue africaniste Richard Fardon (1999) est, essentiellement, une lecture de son œuvre. Directeur du Département de sociologie de l'Université de Montréal, Michel Fournier est l'auteur de deux biographies : Marcel Mauss (1994) et Durkheim (2007). On doit à James Clifford (1987) une biographie pionnière de Maurice Leenhardt qui restitue la complexité historique d'une quête spirituelle et d'une pratique scientifique originale, dans un contexte de lutte coloniale.

2. S'agissant d'auteurs majeurs dont l'œuvre a déjà fait l'objet de multiples commentaires - Durkheim, Weber, Foucault -, l'historien de la sociologie Arpád Szakolczai s'emploie à reconstruire des "expériences fondatrices " : celles en lesquelles s'enracinent sur le plan existentiel, comme leur condition de possibilité, les idées théoriques qui, ultérieurement, marqueront une rupture conceptuelle majeure. Pour cela, il est plus utile de considérer des textes mineurs, en marge des sources scientifiques (autobiographie, journal personnel, correspondance), et de s'attacher à des détails biographiques, à première vue minuscules, mais dont seule la contextualisation historique permet de qualifier leur valeur décisive. Le plus souvent, ajoute l'auteur, il s'agit de moments de dissolution d'un ordre sociopolitique qui rendent problématiques les présupposés implicites de nos manières de penser et d'agir, et exigent, par là même, un intense travail réflexif dont la théorisation ultérieure gardera l'empreinte : en somme, dirions-nous, l'équivalent de l'expérience de terrain pour l'ethnologue (Szakolczai 1995). Reprenant cette proposition fondée sur la notion d'" œuvre de vie ", à propos de Robert Hertz, A. Tristan Riley (2001) montre la richesse de la correspondance privée pour restituer, dans la nouvelle figure d'intellectuel issue de la crise des élites culturelles de la fin du XIX siècle, l'étroite interaction entre programme scientifique, activité politique et préoccupations morales ou religieuses.

3. C’est le cas d'Henri Duveyrier pour les études touarègues (Casajus 2007) et de Maurice Delafosse pour l'africanisme (Amselle \& Sibeud 1998). 
Aussi bien introduisent-elles de nouvelles questions: reconstituer la trajectoire intellectuelle d'un savant transforme-t-il le chercheur en simple historien? Et, si l'on répond par la négative, quelle définition du biographique faut-il adopter, quel genre d'ethnographie faut-il pratiquer pour que celle-ci prenne place dans une anthropologie des savoirs? Soit une récente recherche qui entendait développer les virtualités d'une narration biographique pour éclairer la genèse d'une œuvre à première vue bien connue, celle de l'anthropologue italien Ernesto De Martino (Charuty 2009). Elle conduit, de fait, à repenser la spécificité de la voie italienne de refondation de la discipline, au lendemain de la Seconde Guerre mondiale.

Ernesto De Martino (1908-1965) a dispersé, dans ses écrits, des fragments de récits autobiographiques; il n’a cessé de méditer sur sa trajectoire personnelle et, depuis quinze ans, son œuvre publiée et ses archives sont au centre d'une multiplicité d'entreprises, scientifiques et culturelles, qui transforment le statut de cet auteur. La fabrique d'un " néotarentisme " fait de son œuvre ethnologique un bien culturel pour convertir l'ancienne «terre du remords» - le Salento - en «terre de la mémoire» à des fins d'économie touristique et de reconfiguration des identités régionales. Dans le même temps, philosophes, anthropologues, historiens des religions, médecins relisent, chacun pour soi, une œuvre dont l'unité est, elle-même, problématique. Au milieu des années 1970, les chercheurs italiens inscrivaient De Martino dans l'histoire nationale de l'anthropologie à travers des débats sur la culture populaire, analogues à ceux des années 1950 sur les risques de populisme inhérents à la reconstruction démocratique de la nation. Dix ans plus tard, une approche plus distanciée a produit les premières études sur les conceptualisations empruntées à la philosophie, à l'histoire religieuse, à la médecine, tandis que le classement des archives a conduit à publier une partie des carnets d'enquête, à rétablir des responsabilités éditoriales, à documenter des liens de parenté, de travail et d'amitié avec des figures controversées de la vie savante ou politique.

Outre l'actualisation de l'œuvre au moyen de nouvelles préfaces, ce travail critique permet de reconnaître l'hétérogénéité des champs d'activité et la pluralité des liens intellectuels qui dessinent les espaces sociaux d'une trajectoire individuelle. Mais il ne libère pas le chercheur des attentes sociales et des jeux subtils de pouvoir qui tendent à l'immobiliser dans le rôle de scribe - tour à tour autorisé ou récusé - d'un récit collectif, en concurrence avec d'autres récits collectifs, pour construire une figure d'exemplarité. Conserver une position d'ethnographe, à travers l'immersion dans une fonction sociale de "biographe», requiert de suspendre la naturalité des modèles biographiques qui sous-tendent ces récits et, plus encore, l'évidence de formulations qui renvoient à un sens commun 
historiographique ou au savoir propre à chaque cercle. Somme toute, il s'agit de se soustraire au sens que les uns et les autres vous commandent d'entendre. Défaire le statut et la hiérarchie des documents pour explorer, de l'intérieur, des faisceaux de relations, pour relier entre elles des expériences éclatées entre des lieux et des figures à première vue sans commune mesure, a révélé la complexité, troublante à plus d'un titre - et l'actualité inattendue des questions posées par son interprétation -, du parcours qui précède la part la mieux connue en France d'une œuvre inachevée : la trilogie méridionale, publiée entre 1958 et 1961, qui demande à l'ethnographie d'une altérité chez soi - le catholicisme du Sud - de vérifier quelques grandes hypothèses sur la singularité du christianisme dans l'histoire occidentale.

En effet, l'analyse des traitements rituels des principaux risques de dissolution de la personne n'est que le second moment d'un travail d'hybridation disciplinaire et de reformulation conceptuelle, commencé au milieu des années 1930, pour retourner sur nos propres sociétés un questionnement qui suscite, à chaque pas, une interrogation parallèle sur l'identité scientifique du chercheur. Soit l'introduction à La Terre $d u$ remords, l'œuvre pourtant la plus achevée: tout en faisant siennes les perplexités lévi-straussiennes exprimées dans Tristes Tropiques, tout en usant de formulations proches de celles proposées dans le premier chapitre d'Anthropologie structurale, De Martino se présente tantôt comme un "ethnographe moderne", tantôt comme un historien qui recourrait à l'enquête ethnographique comme une modalité, parmi d'autres, de production de documents. Mais, les historiens de l'école des Annales - Marc Bloch, Lucien Febvre - auxquels il se réfere sont exactement ceux qui, pour Lévi-Strauss, partagent avec les ethnologues une même attention aux dimensions inconscientes de la vie sociale. Et, plus qu'aucun autre chercheur italien, De Martino a longuement réfléchi sur les conditions singulières du travail ethnographique par rapport à toute autre forme de documentation historique. De la même façon, La Fine del mondo, l'œuvre fragmentaire éditée à titre posthume, est traversée par une hésitation sur le champ disciplinaire auquel rattacher l'immense entreprise de penser ensemble trois catégories et trois moments de l'expérience apocalyptique : elle est résolue en s'affirmant comme une "ethnologie réformée " en laquelle on peut reconnaitre, aujourd'hui, une anthropologie de l'histoire (Fabre 2005).

Commencé dès la fin d'études de philosophie, ce travail d'hybridation conceptuelle a, certes, pu s'inscrire dans le cadre universitaire d'une «École de perfectionnement en sciences ethnologiques", rattachée à la chaire 
romaine d'histoire des religions, discipline centrale dans le projet comparatiste de Raffaelle Pettazzoni. Mais, il a pris la forme d'un singulier parcours d'apprentissage qui transforme en "expérience de terrain » des crises existentielles, des engagements et des croyances politiques, et qui prend pour guides d'une formation en autodidacte à l'ethnologie une succession d'initiateurs, largement illégitimes du point de vue académique. Restituer cette conversion ethnographique d'une vie requiert de mettre à distance les narrations biographiques dont De Martino a ponctué son œuvre scientifique, pour en questionner les silences en pratiquant, soimême, une ethnographie dans les archives. Cela consiste, notamment, à lire les diverses correspondances, inédites ou très partiellement publiées, non pour recomposer des influences intellectuelles entre maîtres et disciples, mais pour décrire les espaces relationnels spécifiquement construits par un genre d'écriture qui participe pleinement à la construction de soi des lettrés de ce temps. Au biographe qui suspend, provisoirement, les classements savants entre correspondances privées et scientifiques, entre genres d'écriture intime ou fictionnelle, parviennent alors les échos d'un étrange lexique familial, au sens précis que Natalia Ginzburg (1963) a donné à ce terme. Comme toute langue tombée dans l'oubli, cet idiome poursuit dans la vie du chercheur un destin complexe où se nouent, précisément, quelques-unes des expériences qui feront de lui un ethnographe. De la même façon, accorder une égale attention aux notes de lecture, aux formes initiales de vastes programmes qui seront ensuite remaniés et aux enquêtes qui tournent court, permet d'identifier la valeur d'expérience de terrain que peut acquérir, sous certaines conditions, la constitution de corpus documentaires. Enfin, certains moments de cette œuvre-vie appellent, plus que d'autres, à défaire provisoirement la hiérarchie entre registres d'écriture et lieux de publication : ainsi des très nombreux articles parus entre la fin des années 1940 et la première moitié des années 1950, qu'il s'agisse des "notes de terrain" publiées dans les grandes revues culturelles ou de l'écriture journalistique dans la presse des partis de gauche. Initialement convaincu de la portée subversive de l'histoire des religions, le chercheur s'emploie à transférer cette valeur, dans l'aprèsguerre, à une singulière entreprise de connaissance qui suspend, à nouveau, toutes les distinctions normatives entre militantisme politicoculturel et travail scientifique. Il faut donc se résoudre à "oublier" temporairement la grande trilogie méridionale pour décrire, dans leur positivité, la dynamique concrète des pratiques d'enquête empirique, les postures d'énonciation qu'elles favorisent, les formes du récit de soi qui les accompagnent, les formes de restitution qu'invente, pas à pas, De Martino. 


\section{L'aube d'un monde nouveau}

Dès lors qu'on s'attache à rétablir ces continuités souterraines, apparaissent des coïncidences et des affinités thématiques évidentes entre les problèmes méthodologiques du savoir qui a pour objet la «culture » et l'ensemble d'idées et de langages que l'historien de la littérature Alberto Asor Rosa a appelé le "néoréalisme mouvement " : expression calquée sur une autre, le "fascisme mouvement", introduite par l'historien Renzo De Felice pour distinguer, à l'intérieur du régime mussolinien, une mobilisation politico-culturelle animée d'intentions critiques et progressistes ${ }^{4}$. Parler de "mouvement» signifie, pour l'historien de la littérature, ne pas réduire l'expressivité néoréaliste à un strict moment chronologique, mais reconnaître la similitude des choix culturels qui ont orienté une pluralité de langages, avant et après les années néoréalistes au sens strict (les années 1945-1950 pour le cinéma). De fait, passées les querelles des années 1950 sur le réalisme dans l'art, oubliés les violents débats des années 1970 sur les illusions populistes, l'histoire culturelle italienne, puis française, s'est employée à identifier les éléments communs aux deux principales formes expressives dont l'explosion, dans l'immédiat après-guerre, fut vécue comme une nécessité existentielle par tous les créateurs qui entendaient actualiser ce nouveau pacte réaliste qu'Italo Calvino a justement défini comme «le plus distant possible du naturalisme» (1967 [1947] : 9) ${ }^{5}$. Il suffit, ici, de rappeler brièvement les traits communs à cet intense besoin de «s'exprimer » où le monde entier devait se reconnaître dans la moindre narration locale : ériger en nouveau public des groupes sociaux jusque-là exclus, penser l'œuvre d'art en soi comme un fait politique, redécouvrir l'individu concret à travers le triomphe du narratif, documenter une réalité mineure - la vie quotidienne de microcosmes sociaux - en recourant au récit d'histoires individuelles et de cas singuliers; enfin, s'agissant de la

4. Aujourd'hui reconnu comme un grand historien du fascisme, Renzo De Felice est l'auteur d'une monumentale biographie de Mussolini dont les premiers volumes ont suscité, en Italie, de violentes polémiques. La plus importante a éclaté, en 1975, à propos de la distinction proposée entre fascisme-« mouvement " et fascisme-« régime», pour rendre compte de la mobilisation des classes moyennes émergentes : voir l'introduction d'Emilio Gentile à l'édition française d'un recueil d'articles de l'auteur (De Felice 2000). Alberto Asor Rosa a développé ses thèses sur le triomphe du narratif dans le néoréalisme-« mouvement " à l'occasion du colloque "Cinéma et littérature en Italie, 1943-1953", organisé à Vérone en 1981. On se reportera, également, aux contributions de Giorgio Tinazzi et de Marina Zancan dans Cinema e letteratura nel neorealismo (1990), ainsi qu'aux études rassemblées par Michel Cassac (2004).

5. Préfaçant la réédition de son premier roman - Il Sentiero dei nidi di ragno, publié par Einaudi en 1947 -, Italo Calvino décrit lumineusement l'intention provocatrice qui l'animait lorsqu'il s'associe à l'ensemble de voix périphériques découvrant, alors, la liberté de parler et, surtout, l'apprentissage du récit le plus objectif pour atteindre au maximum de subjectivité. 
fameuse "poétique de l'immédiateté », recourir à de très précises techniques pour passer de l'oralité à diverses écritures de témoignage. Celles-ci construisent la figure du "protagoniste", à partir du modèle fourni par l'expérience vécue de la guerre civile et de la Résistance, pour affirmer, sur le plan esthétique, l'identité du vrai et du beau.

Ces recherches très stimulantes s'arrêtent à la porte de l'atelier ethnologique. Quant à l'historiographie démartinienne, soit elle affirme une affinité générique avec les thématiques sociales de l'art néoréaliste, soit elle souligne les prises de distance publiques du chercheur avec les thèmes les plus diffus du débat d'idées. Dans les deux cas, ce sont les modalités de collaboration de l'ethnologue avec les photographes, puis avec le cinéma documentaire, durant toute la période des enquêtes dans le Sud italien, qui retiennent, pour l'essentiel, l'attention ${ }^{6}$. Or, la mise en pratique de principes largement admis par les anthropologues - restituer aux papiers archivés leur valeur d'actes d'écriture, rétablir l'historicité intérieure d'une existence à partir de temporalités non linéaires, identifier les règles implicites qui modèlent les narrations biographiques, reconnaître la dimension intensément socialisée de l'intimité et la nature relationnelle des identités individuelles, suspendre toute définition normative de l'expérience de terrain et de ses mises en image et en écriture - éclaire des affinités plus essentielles entre la refondation disciplinaire proposée par De Martino et la transformation des goûts et des valeurs que l'on peut, quelles que soient les réserves maintes fois exprimées, désigner par l'expression "néoréalisme mouvement».

Des débats enflammés ont très tôt mobilisé la critique italienne sur l'existence d'une école néoréaliste chez les cinéastes de l'après-guerre, sur ses antécédents dans les années 1930 et sur les crises répétées qui semblaient l'affecter dès la fin des années 1940. Bien plus rares, en revanche, sont les témoignages qui permettent de nous représenter le choc culturel produit sur les spectateurs ordinaires par la rupture de toutes les conventions narratives du cinéma hollywoodien. Il appartient, justement, à Alberto Asor Rosa de ressusciter pour nous, cinquante ans plus tard, au détour d'un magnifique récit autobiographique, L'Alba di un mondo nuovo ( L'Aube d'un monde nouveau »), cette expérience, selon lui, unique dans l'histoire du cinéma - le peuple romain se regardant lui-même en assistant à la projection de Rome, ville ouverte (Roberto Rossellini, 1945) -, dans un quartier populaire où il habitait enfant, à peine quelques mois après la libération de la ville :

6. Elles ont fait l'objet d'enquêtes dans les archives des principaux photographes de De Martino (Faeta 2003; Gallini \& Faeta 1999). 
"Autour de nous, dans la salle, il y avait les mêmes gens humbles, pauvrement habillés, au teint hâve, les joues creusées par la faim, les galoches de liège usées, de pauvres robes de coton léger, les vestes limées, en somme pareils aux personnages qui, à quelques mètres d'eux, racontaient leur modeste histoire sur l'écran; et cette histoire était à peu près la même que celle que les spectateurs dans la salle ou leurs amis les plus proches, leurs parents, leurs voisins avaient racontée peu de temps auparavant dans les rues de Rome, dans les jardins poussiéreux de la périphérie, dans les sinistres immeubles populaires, dans les bars et les petits cafés éparpillés ça et là, avec partout les Allemands et les fascistes armés et menaçants, le règne de l'oppression et de la peur, les souterrains frémissants de révolte, les persécutions et les rafles, la terreur de la torture et de la mort. Ça alors : quand avait-on jamais vu, quand reverrions-nous une chose pareille ? [...] le public dans la salle, c'est-à-dire nous, c'est-à-dire moi, ma mère, notre voisine de l'étage au-dessus, l'épicier de la boutique à l'angle de la rue, la vieille dame dans la loge de la conciergerie, l'électricien qui nous réparait la lumière, le manœuvre des Chemins de Fer avec une jambe plus courte, le retraité qui vivait dans une cave sous l'escalier, nous étions tous là, les yeux écarquillés et la bouche ouverte, à regarder ce qui diable nous était arrivé en ces mois terribles si proches. Et, mystérieusement, les spectateurs semblaient avoir pris conscience de cette situation inédite dès le premier photogramme, l'identification fut totale et immédiate: on entendait dans la salle la respiration du public qui suivait, à l'unisson, le rythme soutenu de l'action; et lorsque la femme brune, au regard intense, qui semblait être sortie du même ventre que la plupart des femmes présentes à ce moment-là - même chevelure noire, rebelle, négligée, mêmes yeux mobiles, noirs, très vifs, marqués par deux grands cernes profonds et, surtout, reconnaissable entre mille, la ligne amère de la bouche, ce signe désespéré, atavique, de l'insurmontable tromperie de la vie -, fut abattue dans le dos par une mitraillette allemande, tandis qu'elle courait désespérément derrière le camion qui emportait son amour, un hurlement de rage s'éleva du parterre; et, à la fin, alors que les lumières revenaient dans la salle, un applaudissement général célébra le prodige de cette identification; et d'un seul geste, à l'unisson, des dizaines de mains munies de mouchoirs, plus ou moins propres, se portèrent aux yeux et au visage pour sécher les fleuves de larmes qui avaient coulé " (Asor Rosa 2002: 315-317, ma traduction).

L'exigence de refonder la langue - parlée, écrite, visuelle - dans ses rapports avec la réalité a, en effet, poussé tous ceux qui ont choisi de documenter des espaces du monde social jusque-là exclus des rhétoriques du régime, qu'il s'agisse de la vie quotidienne d'une rue ou d'un quartier, des métiers du bois ou de la mer, voire - souvenons-nous de cette scène, magnifique et trop peu commentée, du Voleur de bicyclette (Vittorio De Sica, 1948) de la chambre à coucher où consulte une voyante. Or, en littérature comme au cinéma, retrouver la langue parlée au quotidien c'est, en Italie, faire entendre les multiples sonorités dialectales, ce qui pose des questions difficiles à résoudre, comme en témoignent les péripéties du premier, et unique, épisode de La Terre tremble (Luchino Visconti, 1948), justement salué par la critique comme une association paradoxale de réalisme et d'esthétisme. On le sait, en plus de la prouesse technique du rendu, par la photographie, de la profondeur de champ dans le décor réel des maisons 
et les longs plans-séquences pour restituer le temps réel d'actions minuscules, Luchino Visconti avait demandé aux pêcheurs-interprètes du village d'Arci Trezza de transposer eux-mêmes le texte italien de chaque scène, pour qu'en retrouvant leurs mots propres ils puissent rejoindre leur rôle. Mais, après une première projection à Venise où, durant trois heures, le langage des pêcheurs demeurait incompréhensible même au public italien, le cinéaste dut se résoudre à faire doubler les personnages par des acteurs parlant un italien "sicilianisant ${ }^{7}$.

Outre tous les films, devenus désormais des classiques, qui ont donné au cinéma, nous dit André Bazin, non plus seulement une évocation ou une description, mais une écriture de la "continuité vraie de la réalité " susceptible d'en préserver la foncière ambiguïté, il suffit de rappeler les chroniques publiées dans les premiers numéros de Società, les biographies de villages ou de quartiers urbains sollicitées par Elio Vittorini pour Il Politecnico d'inspiration marxiste, aussi bien que le journalisme social de la revue Comunità - liée au mouvement d'inspiration chrétienne d'Adriano Olivetti - qui pouvait faire place, dans le commentaire des photographies, à la mise en forme ritualisée des vies individuelles ${ }^{8}$. Plongé dans ce contexte historique et idéologique, le laboratoire démartinien ne se tourne pas d'emblée vers l'équivalent occidental de ces traitements symboliques des états critiques de l'existence qu'il a réexaminés dans Le Monde magique (1948), et dont Carlo Levi (1947) vient de révéler l'emprise, entre les mains des femmes, au-delà d'Eboli. Il se construit autour d'une première exigence, progressivement élaborée sur le plan théorique : identifier les lieux qui permettent de produire la distinction anthropologique entre le social et le culturel. Autrement dit, reconnaitre des domaines de pratiques structurées par des logiques qui ne se réduisent

7. Inspiré par le grand roman vériste de Giovanni Verga, I Malavoglia, le cycle documentaire La Terra trema devait, initialement, comprendre trois épisodes centrés, chacun, sur un monde social: les pêcheurs, les ouvriers des mines de soufre et les paysans. On lira les deux versions, italienne et sicilienne, du scénario dans Visconti (1951). André Bazin s'est imposé en France comme l'un des meilleurs commentateurs du néoréalisme italien. Je reprends, ici, des éléments de sa belle critique, parue dans Esprit en décembre 1948, et reprise, avec d'autres études, dans Bazin (2007).

8. Ces enquêtes privilégient l'Italie méridionale, des Pouilles à la Sicile, et font un large usage du récit biographique. Ainsi Vittorini réclame-t-il à son père des biographies "authentiques " d'ouvriers des mines de soufre et de paysans pour un nouveau projet : "faire raconter aux gens les plus humbles leur propre vie en une page, et puis utiliser leurs propres mots pour composer une sorte de drame avec des dizaines et des dizaines de personnages, tous tirés du vrai (surtout, j’insiste, prénom, nom et des faits authentiques). Mets aussi la biographie de quelque mort qui puisse être dans cette sorte de drame journalistique la voix des générations passées. Pourrais-tu, aussi, aller chercher des biographies d'hommes du commun dans le passé, en les prenant dans les épigraphes des tombes? Je pense beaucoup à ce genre de travail. Cela devrait être d'une absolue nouveauté " (À son père, 22 décembre 1945, in Vittorini 1977 : 42). 
pas à une fonction de domination idéologique et que, plus tard, De Martino lira en clé symbolique. Comment ce problème de méthode, essentiel pour la discipline, accède-t-il à l'expérience sensible du chercheur ? Une première réponse peut être trouvée dans des écrits techniques, habituellement écartés de la production scientifique du chercheur car étroitement liés à une pluralité de fonctions militantes qui lui font traverser des espaces géographiques et sociaux contrastés.

\section{Réformer la médiation politique}

Tout en assumant des responsabilités de cadre politique au sein du Parti socialiste, De Martino privilégie un travail empirique d'exploration de la différence méridionale en la qualifiant, tout d'abord, sur le plan sociologique. De la distance exotique qui était au centre de ses intérêts intellectuels d'avant-guerre, il passe aux dénivellements sociaux qui prennent valeur de frontières à abolir, au moment de la reconstruction démocratique de la nation. On pourrait dire, en reprenant les différents modèles récemment proposés pour contraster les lieux et les temps d'émergence d'une situation ethnographique au sein de la culture occidentale, que De Martino commence par adopter le modèle De Gérando focalisé, quant à son objet, sur les classes pauvres, et gouverné, quant à son régime d'historicité, par l'idéologie du progrès social (Fabre 2007). Les premières enquêtes dans l'immédiat après-guerre à Bari, à Lecce, à Matera prennent, alors, la forme objectivante du rapport d'expert, ensuite confié à d'autres chercheurs lorsque lui-même s'identifiera comme ethnologue pour traiter d'une altérité féminine et religieuse plus que sociale. Mais, au sein même de cette première caractérisation, De Martino introduit de très neuves exigences qui ouvrent la voie à la reconnaissance de l'arbitraire culturel. Ainsi, l'enquête collective publiée par la revue Quarto Stato, en 1947, reconnaît la nécessité de prendre en compte la différence sexuée pour documenter les conditions de vie et de travail des ouvriers pauvres de la Terre de Bari et, pour ce faire, elle distribue déjà la tâche d'observation entre trois regards : masculin, féminin, médical. C'est aussi en réformateur de la médiation politique que De Martino fait directement connaissance avec ces «Indes de par ici » qui lui apparaitront, dix ans plus tard, comme la patrie élective du tarentisme. Arrivé à Lecce, en février 1950, comme commissaire pour mettre fin à une opposition factieuse dont un précédent commissaire n'a pas réussi à venir à bout, il écoute attentivement les « camarades bourgeois » qui entendent conserver le contrôle de la fédération socialiste. Il se déplace dans leurs fiefs - ces mêmes gros bourgs du Salento qu'il parcourra dix ans plus tard en quête des tarentulées - 
pour identifier les modalités concrètes d'une relation de pouvoir - le "personnalisme»-que l'on ne peut expliquer en clé uniquement économique. Il adresse, sous forme d'études de cas, des rapports à la direction du Parti socialiste, qui esquissent une analyse du défaut de représentation démocratique, en inventoriant les signes minuscules à travers lesquels se construit cet autre régime du lien politique que l'anthropologie méditerranéenne désignera sous le nom de «clientélisme»?.

Durant l'été 1951, c'est le mouvement de mobilisation paysanne pour l'application des premières mesures de la réforme agraire qui pose le problème technique de la transcription d'une langue qui, même lorsqu'elle écarte le dialecte, a ses constructions, ses rythmes, ses vocables, ses sonorités propres. L'enquête sur les conditions de vie des ouvriers agricoles à laquelle De Martino participe dans le Metapontino a été préparée par la CGIL (Confederazione generale italiana del lavoro) de Matera, selon les mots d'ordre issus des Assises pour la renaissance du Sud qui se sont tenues, en décembre 1949, dans toutes les régions méridionales. Or, le chercheur infléchit singulièrement la liste des revendications domestiquées par les instances syndicales, en intégrant un droit à la biographie que n'avait pas prévu le modèle pré-révolutionnaire du cahier de doléances adopté par les militants. Et il propose, comme alternative à la figure chrétienne de victime sacrificielle souvent adoptée pour se raconter, le modèle - médical et scolaire - d'une progression des âges de la vie, de l'enfance à l'âge adulte, afin de faire entrer les braccianti dans le registre du politique, comme citoyens idéalement dotés d'une compétence graphique et d'une continuité biographique. Transcrites et en partie publiées sous forme de lettres dans Vie Nuove - le magazine culturel du Parti communiste -, ces narrations sont encadrées de commentaires où la "culture" se trouve différenciée de ce que les instances d'action syndicale nomment le « social ${ }^{10}$.

Cependant, dans cette période ô combien mouvementée de crise personnelle et professionnelle, les tout premiers séjours à Tricarico précipitent, à l'évidence, le processus de reconnaissance d'une distinction fondatrice qui l'incite à créer à Rome, en marge de l'université, un "centre ethnologique italien ". La condensation d'expériences, émotionnelles et intellectuelles, qui a pris alors valeur de révélation d'un "autre monde", est difficile à documenter car elle participe à la fois de la socialisation d'un tournant biographique personnel et d'une mobilisation, nationale et internationale, d'experts en changement social et culturel. On le sait, depuis

9. De Martino, Lodo sul caso Corciulo, Lecce, 20 juin 1950 (Archives privées d'Aurelio Macchioro). 10. Ces lettres sont conservées dans les Archives De Martino (6.15) et très partiellement publiées dans Vie Nuove avec des photographies du jeune Arturo Zavattini, fils de Cesare Zavattini, qui vient de travailler sur le plateau d'Umberto D. avec Vittorio De Sica (cf. De Martino 1952d). 
l'été 1949, le jeune maire socialiste de Tricarico, Rocco Scotellaro, accueille l'intellectuel, à peine remis d'une hospitalisation de plusieurs mois, ainsi que sa nouvelle compagne, tout comme il introduit les chercheurs américains que lui adressent Carlo Levi ou le sociologue Manlio Rossi-Doria. À la différence des observateurs étrangers, De Martino se soumet à l'apprentissage informel de toutes ces images, ces pensées, ces usages qui composent une "sagesse", par le biais d'une domestication réciproque où l'accès au domaine des femmes est d'autant plus important qu'une grande part de ce qui est transmis comme culture doit être, pense-t-il, cherché dans les rapports entre le catholicisme populaire et ces usages coutumiers auxquels il conserve le nom de "magie ${ }^{11}$. Mais cette conviction ne l'a guère préparé au flux d'émotions cristallisées par l'univers sonore de la Rabata - le quartier le plus pauvre de cet ancien fief du conservatisme clérical -, du «lyrisme sauvage» de ses pèlerinages printaniers à la chapelle de la Madonna di Fonti, des chants aigus ou mélancoliques de ses braccianti, non plus qu'aux étonnantes capacités de distanciation de ceux et celles qui sont à la fois de très conscients sujets historiques et de très habiles acteurs dramatiques. Partager durant plusieurs semaines la vie, au jour le jour, d'un collectif en plein affrontement politique fait immédiatement surgir une question qui nourrit, au même moment, l'écriture autobiographique et poétique de son hôte : comment réintroduire dans le champ d'une culture valorisée cet art de la parole, du mime et du chant et ces répertoires fabuleux si intimement liés à une identité collective dominée?

Jusqu'ici, les nombreux articles publiés dans la presse de gauche commentaient, pour l'essentiel, l'actualité sociale et politique en adoptant, il est vrai, un registre d'analyse absent de l'écriture journalistique: des formes d'expérimentation pour identifier la manière dont les catégories culturelles dominantes se concrétisent dans le faire d'agents historiques individualisés. L'appel à un marxisme créatif affirme l'exigence d'enquêtes empiriques tant pour documenter l'hégémonie que le christianisme romain exerce avec le soutien de l'idéalisme crocien, que pour libérer «le point de vue de l'ouvrier agricole du Minervino » du ghetto des traditions populaires. Ce souci de décrire, de l'intérieur, les formes modernes du pouvoir politico-religieux est directement inspiré par la lecture du Gramsci analyste de la diversité sociale des christianismes, de l'Église comme institution et du clergé comme intellectuel collectif. Mais il s'appuie, en outre,

11. Sur la diversité des modalités d'acculturation réciproque de De Martino et de sa compagne, Vittoria De Palma, durant les premiers séjours en Lucanie, je me permets de renvoyer à Charuty (2000 et $2009: 308-326$ ). 
sur un renversement du point de vue, seul susceptible de défamiliariser les lettrés de l'Église catholique dans le regard occidental. C'est le programme d'ethnologie chez soi qui prendrait pour terrain l'Université catholique de Milan; ce sont les articles publiés dans L'Avanti!, entre l'été 1948 et l'été 1949, pour permettre aux lecteurs d'éprouver, très concrètement, l'étrangeté et la spectaculaire efficacité politique du langage visionnaire mobilisé dans la campagne électorale. En somme, cette écriture d'intervention préfigure un programme difficile d'anthropologie des formes modernes du pouvoir théologico-politique que, de fait, les chercheurs, historiens ou ethnologues, ne seront en mesure de réaliser que cinquante ans plus tard (De Martino 1948b, 1949a et b).

\section{Réformer les ritualités civiles}

À peine établi dans la capitale, De Martino endosse le rôle de réformateur culturel en alternant séjours en Lucanie et voyages en Romagne, pour orienter ici et là la création de nouvelles ritualités civiles susceptibles d'unifier la péninsule, au lieu d'exalter un primitivisme méridional. À cette fin, les collections régionales de traditions narratives deviennent les instruments d'un genre inédit de travail culturel, avant même d'être évaluées comme ressources documentaires pour les ethnographies à venir. Tout comme les cinéastes qui entendent libérer le cinéma de ses éléments professionnalisants pour retrouver la poétique du film que chacun ferait pour soi, De Martino s'emploie à former des collecteurs de répertoires musicaux et narratifs, sélectionnés en fonction de leur valeur "progressiste ". Or, cette volonté de déprofessionnaliser l'étude du folklore conduit, de fait, à redéfinir l'objet même du savoir ethnographique. Tandis que les dialectes et les accentuations régionales de la langue parlée ont fait irruption à l'écran et dans les chroniques, les critères de sélection du populaire et les modalités d'enregistrement qu'il propose dans les réunions de travail organisées dans la région de Ravenne, dans la presse culturelle de Bologne ou, au niveau national, pour les lecteurs du Calendario del popolo - qui, bien sûr, ne sont pas des apprentis-ethnographes mais des militants ouvriers et paysans, ou bien des travailleurs sociaux - sont ceux-là même qu'il s'agira d'imposer à la discipline scientifique. Populaire : c'est-à-dire partagé par une collectivité territorialisée et sociologiquement structurée ; du passé ou du présent; en dialecte ou en italien; selon la plus grande diversité de formes stylistiques et de thématiques parmi lesquelles le travail, les occupations des terres ou des usines, les martyrs de la classe ouvrière, le refus de la guerre. Enfin, à la différence des folkloristes de métier, de très précises recommandations focalisent l'attention des collecteurs 
sur la nécessité de documenter les événements qui donnent naissance aux réalisations vocales, les situations qui les appellent, les rythmes physiques qui leur donnent corps (De Martino 1951a et b).

En somme, la posture réaliste consiste, ici, à suspendre la valorisation exclusive des contenus narratifs de la tradition orale, telle qu'elle fut mise en ouvre dans le régime romantique d'une réécriture littéraire ou dans celui, positiviste, d'une austère reconstruction philologique, pour rétablir l'observation des situations sociales d'énonciation. Les règles de description des performances qui se précisent pas à pas, à travers l'engagement de l'expert culturel pour le renouveau des langages esthétiques, seront quelques années plus tard employées pour tout acte rituel, tandis que la collecte et l'étude des répertoires musicaux seront confiées à un ethnomusicologue. Reconnaître précisément leur moment d'émergence invite à restituer une plus grande complexité à un style d'enquête dont les premiers commentateurs soulignaient, plutôt, la faiblesse théorique ${ }^{12}$. Mais il faut, à l'inverse, rétablir la continuité biographique, des années 1930 aux années 1950, d'une quête plus essentielle de valeurs se substituant au christianisme, pour donner tout son sens à l'engagement épisodique du chercheur dans des formes de cérémonialité qui ont pris en charge, de manière transitoire, la fondation symbolique d'une République encore à venir : celle que Togliatti a qualifiée de démocratie "progressiste ».

De Martino privilégie les langages expressifs qui peuvent soutenir de nouvelles célébrations collectives d'un idéal du vivre ensemble. En ce sens, l'appel de l'intellectuel gramscien pour construire un «folklore progressiste " prolonge, mais en l'inversant de signe, l'activisme de l'avant-gardiste du régime mussolinien pour une "religion civile». Jusqu'ici assimilée à une commune mobilisation juvénile dans un "fascisme de gauche " ou bien singularisée comme un trait de caractère personnel portant au mysticisme, cette adhésion initiale requiert un difficile effort de description - et non un jugement - pour reconnaittre les contraintes et les choix d'un acteur social dans le présent des années 1930, qui n'est ni une victime ni un pur esprit ${ }^{13}$. Éviter de réduire des écrits de jeunesse à des spéculations

12. Voir le dossier rassemblé par Pietro Clemente, "Sul "folklore progressivo" ", in Clemente, Meoni \& Squillacciotti (1976: 115-124).

13. En 1987, dans un séminaire à l'École normale de Pise, l'antiquisant Riccardo Di Donato (1993) met en circulation l'expression "fasciste de gauche" pour qualifier les conceptions idéologiques de De Martino lorsqu'il arrive, à vingt-sept ans, comme enseignant à Bari. L'expression sera constamment reprise ensuite, car elle est devenue, depuis le milieu des années 1970, une sorte de sens commun historique. Ce diagnostic s’appuie sur des archives passées sous silence, des notes rédigées au temps de la vie étudiante à Naples qui ont fait l'objet, quelques années plus tard, sous le tutorat de Marcello Massenzio, d'une thèse de doctorat demeurée inédite. Elles ont été, à nouveau, longuement commentées par le philosophe Gennaro Sasso (2001), .../... 
détachées de toute traduction concrète permet, alors, de contraster un parcours progressif dans les institutions destinées à "refaire " les Italiens avec d'autres parcours possibles. De même comprend-on mieux sa très lente déprise du discours propagandiste en reconstituant, pas à pas, les deux étonnantes pédagogies dont le font, simultanément, bénéficier son futur beau-père, l'archéologue Vittorio Macchioro, et le cercle informel de jeunes libéraux de Bari qui inventent, au cœur même d'une ville promue par le régime au rang de moderne capitale régionale, de très précieuses techniques de résistance intellectuelle.

Comment rétablir toutes les incertitudes du jeune historien des religions s'efforçant de promouvoir un "fascisme-religion", si ce n'est en distinguant plusieurs voix dans ces traces écrites - notes manuscrites, articles de journaux, correspondance ${ }^{14}$ - qui sont moins des pensées, que des actes de parole exigés par un régime soucieux que l'on parle sans cesse de lui sur un ton messianique? La voix de l'instructeur qui tente de faire siennes les convictions d'un camerata n'est pas celle de l'intellectuel critique qui évalue les aspirations d'un pouvoir politique à la légitimité d'une autorité religieuse, ni celle du jeune chercheur soucieux de caractériser les spécificités de "la religion » à partir d'institutions historiquement documentées. De la même manière, comment décrire la très subtile rééducation, sentimentale et civique, qui a produit la conversion anti-fasciste de la fin des années 1930, si ce n'est en lisant correspondances familiales et chroniques individuelles - absentes des archives scientifiques de l'auteur pour identifier les modalités d'un très précis apprentissage d'autres règles du vivre ensemble, à travers la dynamique interne de formes de sociabilité obstinément opposées au "langage meurtrier " et à la disparition du soi dans les mobilisations de masse ${ }^{15}$ ?

Les deux registres - subjectif et politique - de ce renversement entraînent, nous y reviendrons, d'autres remaniements sur le plan des objets intellectuels. Le plus immédiatement perceptible est celui qui s'efforce, difficilement,

[Suite de la note 13] non plus en termes de «fascisme de gauche » mais d'une « exaltation mystique » et d'un "hyperfascisme" qui pourrait, aussi bien, se retourner en son contraire. L'inscription au PNF (Partito nazionale fascista) en 1930 et, surtout, à la Milice volontaire en 1932 qui fait accéder De Martino au statut d'instructeur, est documentée par Valerio Severino (2002).

14. Ce sont, pour l'essentiel, des notes dactylographiées que l'on peut dater de la fin des années 1920 à la moitié des années 1930 (Archives De Martino 1. 06), des articles publiés dans le journal L'Universale et les premières années de la correspondance avec Vittorio Macchioro (Fonds Macchioro, Département des sciences de l'Antiquité et d'italianistique, Université de Trieste).

15. Outre le travail des éditeurs Laterza avec Benedetto Croce, il faut surtout décrire les modalités informelles de la résistance intellectuelle des jeunes libéraux de Bari, parmi lesquels Fabrizio Canfora et Michele Cifarelli, ainsi que le rôle du méridionaliste Tommaso Fiore dans l'initiation de De Martino à la diversité culturelle des Pouilles, dès la fin des années 1930 (cf. Charuty 2009 : 145-174). 
de soustraire à l'emprise des idéologues du régime un vaste programme de philosophie de l'histoire, centré sur les échanges entre le politique et le religieux dans l'Occident chrétien. Or, à la surprise du biographe, les acquis conceptuels qui orientent ce programme se transforment, entre 1943 et 1945, en mots d'ordre politiques susceptibles, croit-on, d'orienter dans le sens d'une "religion de la liberté » l'activité de résistance clandestine en Romagne. Mettre en perspective la succession des engagements existentiels pour une religion civile qui prend, tour à tour, le visage de la justification idéologique d'une dictature, d'un prophétisme révolutionnaire d'inspiration crocienne, enfin de l'entrée dans une seconde résistance d'inspiration marxiste, ne permet pas seulement de rétablir la cohérence d'une trajectoire en se mettant, comme le suggère le biographe d'Antonia Byatt, à l'écoute des leitmotive d'une vie. Apparaît plus nettement l'originalité, dans la bataille culturelle qui transforme l'Italie de l'aprèsguerre en laboratoire politique, de ce que nous appellerions aujourd'hui une anthropologie "participative" où les questions théoriques sont d'abord traitées sur le mode performatif.

Bien avant que ne soit reconnue, sur le plan conceptuel, l'existence de formations de compromis en lesquelles s'affirme une autonomie "relative " du symbolique, l'incitation à valoriser des usages coutumiers et des narrations à valeur progressiste, alors que le Vatican s'affirme comme un redoutable acteur politique, prend, implicitement, en charge une revendication et une injonction: « le droit à la culture contre le devoir de religion ». Et, somme toute, il paraît vain de chercher à fixer une date de naissance pour ce qu'une communauté de métier reconnaîtrait, aujourd'hui, comme une enquête ethnologique. Si, à la différence du travail en Romagne plutôt tourné vers la formation de collecteurs du répertoire narratif, celui qui démarre en Lucanie est plutôt axé sur les marquages rituels du biographique, là aussi «hommes, femmes et enfants se sont approchés des micros ». C'est en journaliste que Marcello Venturoli rejoint l'expédition de l'automne 1952 et Diego Carpitella n'est pas encore le grand ethnomusicologue qu'il va bientôt devenir, mais assume les fonctions de critique musical pour les quotidiens et les magazines de la presse de gauche. En même temps qu'est recueillie une grande partie de la matière ethnographique élaborée, quelques années plus tard, dans Italie $d u$ Sud et magie, la métamorphose culturelle de répertoires expressifs territorialisés et historicisés s'appuie sur une double mise à distance, pédagogique et esthétique, qui ne peut que se tourner vers les langages sonores et visuels du néoréalisme - quitte, là aussi, à les réformer pour s'opposer, tant au réalisme soviétique, qu'au folklorisme du régime mussolinien. Telle est la fonction des présentations abondamment 
illustrées données aux magazines culturels; celle des programmes radiophoniques pour "secouer une certaine paresse auditive" en alternant la mélancolie des chants de travail et la frénésie d'une "chaste orgie paysanne " où fait retour une voix féminine "crue et métallique » ${ }^{16}$; ou celle de la réflexion proposée par le chercheur en réponse à l'enquête sur le réalisme lancée par la revue Filmcritica en décembre 1952. Constatant que les surprenants contrastes d'archaïsme et de modernité qui singularisent les mondes ruraux sont moins familiers aux cinéastes que la vie des milieux populaires urbains, il leur est conseillé de se donner une formation ethnographique pour mieux transformer en patrimoine culturel les ressources symboliques - rites biographiques et récits mythiques - des sociétés paysannes ${ }^{17}$. Et, si l'on en croit l'enthousiasme du chroniqueur de Mondo Operaio, à peine rentré de Lucanie, De Martino tient lui-même le rôle de ce cinéaste ethnographe, avec l'aide du travail photographique de Franco Pinna et musicologique de Diego Carpitella, au grand congrès sur la culture organisé à Bologne en janvier 1953. Le conférencier est « un expert de grande classe " du monde de la magie et du mythe; les images photographiques, en "projection mouvementée ", des rites biographiques et des occupations de terre sont splendides; mais ce sont surtout les chants qui l'émeuvent:

"Une berceuse très douce et rude; un jeu de "conta" entre garçons splendides dans la tessiture des voix; une lamentation funèbre maternelle peut rivaliser avec les lamentations classiques de la musique jazz (et ici la comparaison avec les nègres d'Amérique n'est pas irrévérencieuse pour les gens de Lucanie); un jeune, parti en France au maquis, aux prises maintenant avec une chanson villageoise d'où émerge, brusquement, le motif bien connu et très populaire d'un chant de partisans, souvenir de ces années en terre étrangère; enfin un chant pour la mort d'un âne, très brillant et très déployé, que l'on a envie de réécouter d'un bout à l'autre à peine est-il terminé " ${ }^{18}$.

De la défiance à l'égard du réalisme soviétique témoigne, également, la contribution du chercheur pour aider ce qu'on appelle, alors, le «théâtre de masse " à surmonter la crise diagnostiquée par la commission culturelle du Parti communiste. Encore faut-il, pour en mesurer la portée, s’interroger

16. L'expression «secouer une certaine paresse auditive» est de Diego Carpitella (1952: 739); des fragments du programme radiophonique, préparé et commenté par De Martino, et diffusé le 9 juillet 1953, sont disponibles sur internet (http : //demartino.icbsa.it/).

17. Le film de Giuseppe De Santis, Non c’è pace fra gli ulivi, lui paraît restituer de manière artificielle le mélange d'archaïsme et de modernité propre aux sociétés rurales. Il est plus élogieux pour La Terra trema, mais s'étonne de l'absence de tous les rites biographiques, du répertoire des chants et des croyances concernant les animaux (De Martino 1952f).

18. Giuseppe Bartolucci, "Il congresso della cultura popolare a Bologna», Mondo Operaio, 24 janvier 1953 (in Charuty 2009: 343-344). 
sur la nature exacte des cérémonialités que cette expression désigne, en déplaçant le regard du spectacle lui-même à sa préparation. Le récit autobiographique de Luciano Leonesi (1989), qui a poursuivi à Bologne jusqu’à la fin de 1952 le travail théâtral commencé par le premier metteur en scène Marcello Sartarelli, décrit avec beaucoup de vivacité les répétitions du spectacle de Bologne, Sur le chemin de la liberté. "Les protagonistes dans la vie " sont invités à se raconter eux-mêmes sur une scène, c'est-à-dire à se distribuer entre divers groupes - les fascistes, les Allemands, les partisans, les ouvriers, les ouvrières des rizières. Un épisode de la libération de Bologne sert à territorialiser la transformation en spectacle des événements dramatiques du passé proche. Ainsi, se raconter soi-même signifie entrer dans un drame collectif où les événements tragiques de l'histoire récente sont convertis en un récit mythique dans lequel les forces du Bien et du Mal s'affrontent pour donner naissance à cette nouvelle société qui se présente comme une République progressiste. Répété dans les villes où les fédérations communistes sont les plus puissantes, le récit est particularisé par le choix des protagonistes et par celui des épisodes locaux d'affrontement entre la cruauté des ennemis et l'héroïsme partisan. Les commentaires de L'Unità opposent ce théâtre aux liturgies de l'Année sainte. Mais n'estce pas, justement, cette secrète dimension de rite de fondation de la République qui risque de transformer sa répétition en symbolisme vide? En ce moment d'incertitude, De Martino entreprend l'exploration des ressources locales du théâtre traditionnel et dialectal en vue de structurer un Théâtre national populaire, sur le modèle français de Jean Vilar, mais avec d'autres perspectives: intégrer au répertoire classique les mêmes thèmes à valeur progressiste proposés pour les chants et les chroniques.

\section{Autoportrait de l'ethnologue protagoniste}

On peut reconduire au même climat politico-culturel du tournant des années 1950 l'élaboration, pour l'anthropologie nationale, de la charte mythique de l'ethnologue de terrain, selon les deux lieux - interne et externe - de confrontation avec l'altérité culturelle. Exactement contemporain du congrès de Pérouse qui a permis au mouvement cinématographique de prendre conscience de soi comme phénomène culturel, comme style et comme programme (24-27 septembre 1949) ${ }^{19}$, le fameux manifeste "Autour d'une histoire du monde populaire subalterne", qui convie tous les intellectuels de gauche à devenir "protagonistes" d'une décolonisation culturelle, amorce une complexe narration biographique pour mettre sa vie sous le signe d'une coïncidence

19. Les actes sont publiés par Umberto Barbaro (1950). 
idéale entre l'intellectuel organique et l'ethnologue de terrain. Ces gestes répétés de définition publique de soi participent d'une valorisation collective du témoignage et du récit de vie, que l'on décline à titre de thérapie sociale sous des formes très variées, pour accompagner, malgré l'échec électoral des partis politiques de gauche, la transition de l'Italie vers une démocratie progressiste. L'incitation à assumer le "je " du récit à la première personne ne vaut pas seulement pour transformer les dominés en acteurs historiques ${ }^{20}$. Quelques intellectuels - tels Ruggero Zangrandi, Bianchi Bandinelli, Franco Fortini - se sont, les premiers, livrés à un exercice d'analyse rétrospective pour rétablir une continuité subjective, par-delà le ventennio mussolinien et les années de guerre civile, entre les illusions du passé et les exigences du présent. Mais De Martino répond d'une singulière façon à cette sollicitation.

Cet autoportrait propose aux lecteurs de Società et de Nuovi Argomenti le récit d'origine d'un ethnologue autochtone qui retourne sur soi le questionnement habituellement réservé aux "autres", à partir d'une constellation d'expériences de dépaysement par la pensée et l'action: l'adhésion à l'historicisme crocien, le rejet du paradigme naturaliste dominant l'ethnologie coloniale, le militantisme dans le Mezzogiorno et la lecture de Carlo Levi. À y regarder de plus près, on peut y distinguer l'hybridation de trois modèles biographiques alors disponibles: celui, marxiste, de la critique et de l'autocritique imposé, comme l'a récemment montré Mauro Boarelli (2007), par le Parti communiste à tous les militants formés dans les écoles du parti; celui de l'examen de conscience de matrice chrétienne ; enfin, celui, déjà présent chez Carlo Levi, d'une figure de médiation traditionnelle entre les mondes humains et non humains.

Le ton est celui d'un prophétisme ethnologique qui laisse apercevoir, en raccourci, tous les ingrédients qui vont permettre aux acteurs culturels de la société civile d'accompagner une refondation disciplinaire placée d'emblée, avec le financement de partis politiques et d'institutions culturelles, sur la scène intellectuelle avant même d'être constituée au sein d'une communauté scientifique. À travers un cumul de légitimités et de thématiques, De Martino invite à entrer en une nouvelle résistance, en s'employant méthodiquement à une fonction de passeur culturel entre des groupes sociaux, des publics et des générations qui s'ignorent, des politisations antagonistes. Et les questions agitées dans le débat public débouchent sur des interrogations centrales pour la discipline: qu'est-ce qu'une culture? Qu'est-ce qu'une mythologie? Qu'est-ce qu'une religion? Qu'est-ce qu'un millénarisme?

20. Sur ces usages militants du récit biographique, je me permets de renvoyer à Charuty (2000). 
La représentation d'une posture de l'écoute, encore aussi inhabituelle en ethnographie qu'en politique, vient compléter la composition de ce nouveau protagoniste. On ne saurait trop vite la considérer comme une description des pratiques réelles d'enquête. Il s'agit de donner à voir et à entendre des rencontres, inscrites dans la matérialité des corps et des voix, de leurs attractions et de leurs répulsions, évidées de toute analyse psychologique: l'écriture si singulière des textes donnés aux nouvelles revues culturelles, où l'auteur apparaît en quête d'un style qui imite l'absence de style, assume délibérément la rupture avec la langue académique pour une composition expressive qui se veut la forme nécessaire d'un rêve de démocratie directe. Équivalente au principe «tout est racontable " qui préside à l'écriture filmique et aux narrations de témoignages, la forme adoptée dans Note lucane - le premier texte issu de l'expérience initiale de Tricarico - conjugue, en un montage analogue au découpage cinématographique en longs plans-séquences, un éventail de "faits » qui appartiennent, pour nous, à des régimes distincts de la connaissance du social : des données chiffrées sur les conditions d'habitat et de travail, avec la transcription d'un carnet de comptes et d'une lettre; une séquence quasi panoramique de violence populaire avec ses inventions langagières ; des dialogues improvisés de satire sociale; une scène de théâtre populaire avec les rôles et les jeux de scène; les conditions de création et d'exécution d'une chanson que l'observateur a vue naître sous ses yeux; les propos échangés au cours de moments de joyeuse convivialité ; une réflexion sur la distinction paysanne entre le Jésus-Christ des opprimés et l'Église des puissants; une scène mouvementée d'affrontement entre l'évêque et une militante communiste. "Après ma rencontre avec les hommes de la Rabata", conclut De Martino, retrouvant exactement la définition proposée par l'écrivain et scénariste Cesare Zavattini pour caractériser l'art néoréaliste - " un art de la rencontre » - qui exigeait, selon lui, l'invention d'une nouvelle forme expressive : des "livres néoréalistes " où "le cinéma devient livre ${ }^{21}$. Nous sommes, ainsi, invités à considérer comme également pertinents tous ces registres d'action et ces formes de restitution de l'expérience de terrain à travers une écriture qui apparaît comme l'équivalent, dans le champ de l'anthropologie, de cette poétique de l'immédiateté, faite de réceptivité pure sans médiation, de descriptivité apparemment spontanée qui sont, au cinéma et dans la littérature, le résultat de choix

21. Ce projet sera réalisé à Luzzara, son village natal, avec Paul Strand auquel il explicite son idéal d'enquête: "Je voudrais réussir à faire sortir la réalité la plus réelle possible des mots, des confidences des habitants, rencontrés dans la rue ou chez eux ou au café, questionnés longuement ou brièvement, avec effort ou avec facilité ", Cesare Zavattini à Paul Strand, 13 janvier 1953 (in Gualtieri 2005). Sur la construction visuelle de la Lucanie par les photographes, outre les études de Francesco Faeta, voir les monographies rassemblées par Ferdinando Mirizzi (2009). 
stylistiques très précis pour produire des effets de réalisme fondés sur des effets de présence ${ }^{22}$. Trois ans plus tard, enrichi d'une expérience qui contient déjà, en raccourci, tous les thèmes de la grande trilogie méridionale, le journal de terrain fictif - Notes de voyage - restituera le flux de conscience de l'ethnologue, explicitera ses questions intérieures et ses associations d'idées comme, dans les autres langages expressifs, se trouve modifié le rapport entre le personnage et son milieu. D'une part, on donne au personnage une épaisseur objective en l'immergeant dans les choses et, de l'autre, le glissement vers le regard subjectif nous restitue un paysage mental fait de projections d'états de conscience selon le principe, identifié par Gilles Deleuze, de suspension de l'image-mouvement pour produire un rapport onirique à la réalité en même temps qu'est inventée la conversation comme acte pur de sociabilité (Deleuze 1985).

Les chercheurs italiens qui, au milieu des années 1970, ont entrepris d'écrire l'histoire nationale de la discipline, à partir des débats du moment sur les cultures populaires, ont considéré avec embarras ces mixtes impurs entre travail syndical, enquête sociale, ancienne collecte folklorique et future histoire orale. Posture populiste et retard scientifique de l'ethnologie italienne, isolée durant vingt ans de régime fasciste: tel fut le diagnostic souvent posé sur cette gestation mouvementée de la part de l'œuvre démartinienne qui retenait, alors, exclusivement l'attention. Pourtant, aussi bien le registre héroïque que le nouveau pacte réaliste à valeur politique pour restituer "la continuité vraie de la réalité », dira André Bazin - "l'immédiateté et la spontanéité de la vie quotidienne », explique De Martino aux auditeurs de la RAI -, ont été précédés et préparés par de longues années d'un apprentissage systématique à l'ethnologie, plus secret sinon plus solitaire, ponctué d'une diversité de façons d'écrire dont il faut restituer, au plus près, les potentialités et les effets sur les manières de penser.

\section{Exercices de style}

Sous la conduite épistolaire de l'africaniste Bocassino, inspecteur au musée Pigorini et représentant accrédité de cette école de Vienne dont, pourtant, il a violemment récusé les présupposés métaphysiques dans un premier livre, Naturalismo e storicismo in etnologia (1941), le jeune chercheur en histoire des religions a entrepris, au début des années 1940, l'examen de tous les débats canoniques de l'anthropologie. Toujours en quête d'une « religion civile» dont les virtualités pourraient être documentées par une ethnologie historiciste, il dresse l'état des lieux des débats sur les formes de

22. On en trouvera une description renouvelée dans Casetti \& Malavasi (2003). 
pensée et de vie religieuse des peuples "primitifs", à partir des enquêtes empiriques qui les ont renouvelés dans les années 1920-1930, avant de se concentrer sur l'ensemble des phénomènes réunis sous le nom de «magie». À la différence de l'anthropologie anglaise qui inscrit l'analyse de la magie dans les situations ordinaires, en laissant de côté les spécialistes, mais semblablement à la tradition française inaugurée par Hubert et Mauss, il s'est tourné en priorité vers l'individu magicien en sélectionnant des œuvres ethnographiques qui, quelles que soient les écoles et les théories auxquelles elles se rattachent, sont marquées par un remarquable degré de familiarité de l'enquêteur avec les sociétés décrites. Ainsi, l'œuvre monumentale consacrée par le Père Martin Gusinde aux Indiens de Terre de Feu repose sur un long parcours d'acculturation indienne qui favorise une intimité culturelle aussi grande que celle de l'écrivain-explorateur Knut Rasmussen, lequel a rassemblé sur les pratiques chamaniques des Inuit des données dont la qualité a découragé de nouvelles enquêtes jusque dans les années 1970.

Cette exploration médiatisée par les livres a produit des centaines de pages de notes de lecture, soigneusement conservées et disponibles aujourd'hui dans les archives. Une sorte de bibliothèque portative qui privilégie la description du "chaman en action", soit l'exercice d'une compétence spécialisée : les interventions improvisées ou réglées comme un cérémonial, les démonstrations ludiques ou spectaculaires et les consultations particularisées, les instruments, les pratiques oniriques, la lecture des signes, les modes d'accès à la fonction et l'acquisition des esprits auxiliaires, les théories concernant la personne, les réactions émotionnelles des indigènes et de l'ethnographe. Â chaque fois, traduisant mot à mot des récits ou résumant un ensemble de données exposées par l'auteur, De Martino prend soin de préciser s'il s'agit d'informations directement obtenues par l'ethnologue, ainsi que l'identité de son ou ses interlocuteurs. De même, il apprend à distinguer les différents degrés d'adhésion ou de scepticisme, les doutes qui saisissent l'observateur, ses moments de distance critique ou, au contraire, d'implication personnelle. Mais il note, aussi, les savoirs divinatoires plus diffus et les expériences singulières que l'on rapporte à l'enquêteur pour authentifier des formes de communication extralinguistique et l'attribution d'intentionnalité à des catégories d'être qui, pour nous, ne sont pas des personnes.

Insensiblement, l'activité de notation associée à celle de la traduction de l'anglais ou de l'allemand en italien se transforme en écriture personnelle. De Martino commence par résumer des descriptions, sélectionner des aspects de la situation vécue, prolonger l'analyse par des réflexions ou des hypothèses différentes de celles de l'auteur. Très vite, l'activité de 
transposition dans une autre langue devient le support d'un intense rapport d'implication personnelle dans l'interaction ethnographique, par un double mouvement d'identification à l'enquêteur - qu'il soit missionnaire, explorateur ou ethnologue professionnel - et à ses interlocuteurs privilégiés. Une identification imaginaire qui oriente l'attention critique, moins sur l'analyse des institutions sociales, que sur les modèles psychologiques auxquels les auteurs ont recours pour rendre compte de toutes ces expériences sensibles qui contredisent le sens commun.

Ce faisant, De Martino se livre à des exercices de style pour expérimenter une pluralité de langages descriptifs qui coexistent sur le papier, comme ils vont, durant plusieurs années, coexister dans sa tête. La première réécriture qui a, bien souvent, les accents d'une parodie sérieuse, consiste à conformer ces ethnographies à l'hypothèse d'une faculté extra-sensorielle particulièrement développée chez les peuples dits primitifs, thèse qui est l'exact opposé, sur le plan structural, de celle d'une révélation primitive défendue par l'anthropologie missionnaire. La seconde, qui a laissé beaucoup plus de traces dans les archives que dans les articles ou livres publiés, privilégie une métapsychologie infiniment plus complexe, celle de Pierre Janet, qui permet de faire place aux structures sociales, linguistiques, cosmologiques que mettent en jeu les cures rituelles. C'est en pratiquant, tour à tour, ces deux idiomes descriptifs et analytiques, qu'à la manière d'un écrivain De Martino se défait de la langue technique de ces ethnographies, pour élaborer son propre style descriptif et ses propres conceptualisations.

Somme toute, le jeune chercheur pratique une ethnographie au second degré et non une anthropologie de cabinet, en inventant une manière toute personnelle d' "être sur le terrain". Nous savons, aujourd'hui, combien l'anthropologie anglaise, dans la seconde moitié du XIX siècle, était soucieuse de la qualité des données ethnographiques de première main ; combien les chercheurs de l'école de Cambridge, parmi lesquels Malinowski arrive en dernier, ont pratiqué l'étude intensive de terrain ; et combien cette expression polémique - armchair anthropology - relève du désir d'apparaître comme le premier ethnologue de terrain. Le nouveau pacte ethnographique proposé par Les Argonautes du Pacifique est tributaire d'une construction littéraire de la présence participante, alors que l'activité effective de Malinowski réside essentiellement dans l'observation et dans la sollicitation de récits (Stocking 1983). Dans un autre contexte, lorsqu'on considère le passage à l'ethnologie américaniste du médecin Paul Rivet, durant sa mission au service de l'anthropologie physique, il apparaît qu' "être sur le terrain" parmi les Indiens du Riobamba, au début du $\mathrm{XX}^{\mathrm{e}}$ siècle, ne signifie pas conduire des enquêtes directes au sens où nous l'entendons aujourd'hui, mais consiste à enregistrer les savoirs acquis par 
une diversité d'acteurs sociaux, à travers leurs rapports d'intérêt et de pouvoir avec les populations indigènes (Laurière 2008). En somme, on peut "être sur le terrain» et rester relativement à distance des pratiques qui seront ensuite décrites comme si l'observateur y avait participé - Malinowski -, ou mettre en acte les mêmes médiations que celles utilisées par un armchair anthropologist - Paul Rivet. En observant la formation que De Martino se donne au début des années 1940, on peut encore identifier un autre type d'expérience ethnographique: rester physiquement chez soi, à Bari, et vivre sur le mode imaginaire - qui ne veut pas dire irréel - la confrontation avec une pluralité de cosmologies et de registres d'action, à travers un intense rapport de lecture/réécriture d'œuvres-monde, elles-mêmes produites dans une étroite intimité avec les univers culturels décrits.

Ce long apprentissage de techniques de description centrées sur la dimension performative des expériences contre-intuitives, et non sur leur dimension sémantique comme dans le structuralisme lévi-straussien, est évidemment disponible en tant que savoir incorporé lorsqu'après les deux années de guerre vécues en Romagne, l'ethnologue De Martino retourne à la maison, à tous les sens du terme. On objectera, sans doute, que cette compétence ne peut se substituer à l'expérience directe, surtout s'agissant de toutes ces formes de résolution des situations de malheur individuel ou collectif qui sont, depuis le début, au centre de l'anthropologie démartinienne. Expérience à laquelle, on le sait, Jeanne Favret-Saada (1990) a donné un statut épistémologique très précis à travers l'expression "être affecté ». Mais il suffit de suspendre quelques-uns des principes de classification qui président à la mise en archives de vies savantes et de faire place en elles, comme en toute autre vie, à une pluralité de manières désaccordées de satisfaire le besoin de sens, pour voir apparaitre, dans cette trajectoire individuelle, un exact équivalent de ce moment originaire - où prend naissance le désir de savoir de l'ethnologue - intensément vécu mais seulement partiellement reconnu.

\section{Les expériences domestiques de l'altérité culturelle}

Cette expérience, qui appartient à la vie domestique d'avant-guerre, est inscrite dans la période de plus grande intensité d'une relation de parenté par alliance avec l'archéologue Vittorio Macchioro, inspecteur au Musée archéologique de Naples, lorsque De Martino épouse sa fille, Anna, en 1935. Plusieurs études ont, déjà, partiellement reconstruit les intérêts scientifiques de ce parent culturel aux multiples identités sociales avec lequel l'étudiant, puis le jeune enseignant de philosophie noue, durant 
dix ans, une intense relation affective et intellectuelle ${ }^{23}$. Il était, certes, éclairant de considérer le travail effectué par l'archéologue pour identifier les idées, les questions, les choix analytiques que son œuvre novatrice a pu suggérer au futur analyste des magies primitives et du tarentisme. Mais pourquoi écarter avec embarras - voire assimiler à une irrationalité pathologique - l'expérience vécue du réformateur religieux que ce juif assimilé s'est obstinément efforcé de devenir au sein du catholicisme moderniste, puis du néo-évangélisme protestant, et de faire authentifier sur le mode académique, à travers une reconnaissance institutionnelle comme historien des religions? Reconnaissons, plutôt, que la notoriété scientifique internationale obtenue, au début des années 1920, avec l'étude des fresques de la Villa des Mystères à Pompei s'est progressivement effritée avec d'autres ultérieures sur les relations entre la mythologie orphique de la passion dionysiaque et le christianisme paulinien, largement surdéterminées par l'engagement de Macchioro dans le développement, en Italie, d'un mouvement néo-évangélique. Ces études, parfois confondues avec l'activité de publiciste confessionnel, lui ont fermé la porte des universités italiennes. Mais elles lui ont ouvert, dans les années 1930, celles des collèges théologiques américains en même temps que celle d'une fonction épisodique d'attaché culturel en Inde, au service du tout nouvel Institut pour le Moyen et l'Extrême-Orient. C'est au cours de ce séjour de deux ans en Orient que se noue avec le jeune De Martino, dont il vient de guider les premiers pas dans la recherche pour son diplôme de fin d'études universitaires, une étrange relation épistolaire dont la valeur formatrice pour le futur ethnologue ne se laisse percevoir que si l'on s'efforce de restituer en ethnographe les expériences et les exigences spirituelles de son beau-père.

À la surprise du biographe, ce sont les acquis de précédentes recherches sans liens immédiats avec le laboratoire démartinien qui permettent de maintenir, à travers les archives de vies entremêlées, une exigence de description analytique. Explorer la relation à distance qui lie un jeune et ardent avant-gardiste de la Révolution nationale à un intellectuel à contrecourant des savoirs autorisés fait, tout d'abord, apparaître une forme singulière de "cure par correspondance ", imposée à titre de rééducation politique. Elle évite le conflit d'idées pour restaurer un espace social d'expression de la subjectivité, en se recentrant sur ce que l'archéologue perçoit comme la plus grande menace du fascisme sur le plan culturel : la dissolution de l'identité subjective. Parallèlement, Macchioro adresse à sa famille, sous forme de correspondance collective, une chronique

23. Elles sont rassemblées par Riccardo Di Donato qui a publié, en deux temps, une sélection de la correspondance échangée entre Macchioro et De Martino (Di Donato, ed. 1993 et Di Donato 1999). 
hebdomadaire de sa découverte de l'altérité indienne. Au nombre de ses principaux centres d'intérêt : l'intense activité des temples et des pèlerinages, les réalisations sociales du néohindouisme, l'apprentissage du yoga que les intellectuels nationalistes lui désignent comme le cœur de la sagesse hindoue, enfin ses nombreuses consultations de devins pour mener à bien une mission professionnelle de plus en plus confuse. Plongé dans la quête des précurseurs de cette religion civile qu'il s'efforce de reconnaître dans la "mystique fasciste ", De Martino paraît, d'abord, indifférent à l'exotisme oriental. Il lui faut commencer à se déprendre de ses premières croyances politiques pour que la trajectoire existentielle de son beau-père puisse prendre place dans sa propre quête, existentielle et conceptuelle, d'une "autre" religion qui, cette fois, reprend à nouveaux frais l'interrogation que se renvoyaient, au début du siècle, la philosophie et l'anthropologie, sur les formes élémentaires de l'expérience religieuse et les propriétés de la pensée primitive.

L'espace biographique qui s'ouvre à nous témoigne alors d'une déroutante ferveur religieuse que Macchioro adresse spécifiquement à son gendre pour en faire un disciple et qui semble exercer de non moins déroutants effets de contagion. Elle alimente l'imaginaire, déconcertant chez un lettré soucieux d'argumentations rationnelles, d'un incessant combat contre les puissances du Mal où il occupe, tour à tour, les places de victime et de sauveur dans l'exercice d'une fonction de guide prophétique d'un « christianisme intégral ». Ce langage est, bien sûr, à contextualiser sur le plan sociologique dans la cartographie des innovations religieuses alors actives dans la péninsule. Mais pour en décrire son fonctionnement empirique, nous disposons, heureusement, de la minutieuse chronique que Vittorio Macchioro a tenue tout au long de sa vie; elle éclaire, du dedans, cette étonnante aventure spirituelle ${ }^{24}$.

Dix ans avant le voyage en Inde, pour résoudre la crise religieuse qui a suivi son retour de la Grande Guerre, Macchioro a pris l'habitude de recourir à une grammaire providentielle pour lire sa vie au jour le jour, en usant d'une multiplicité de techniques divinatoires qui peuvent se substituer ou s'ajouter les unes aux autres, au gré de ses migrations entre groupes catholiques et protestants à l'intérieur de la péninsule. L'exploration de ces très précieuses archives permet, notamment, de parcourir quelques-uns des cahiers sur lesquels Macchioro enregistrait les "Réponses" de Dieu, c'est-à-dire des versets de la Bible précédés du motif de la consultation, de la minutieuse description des techniques de sélection et de lecture, et suivis d'un commentaire qui assure l'adéquation de la réponse à la situation.

24. Correspondances et cahiers sont conservés dans le Fonds Macchioro, Université de Trieste. 
On y apprend également que pour "faire parler les évangiles", en suspendant l'intentionnalité du lecteur, il faut, en outre, se mettre à l'écoute de la parole inspirée de femmes expertes en "voyance» qui vérifient la parole de Dieu. Tels sont les recours les plus constants, mobilisés dans tous les moments d'incertitude dans la vie familiale et professionnelle, dans l'exercice de la fonction paternelle qu'il assumera durant quelques années sur le jeune couple d'Ernesto et d'Anna, et dans les conflits, parfois aigus, qui impliquent les complexes relations affectives entre le couple des parents et celui des enfants. Au lieu de les écarter a priori comme relevant de la seule trajectoire existentielle de Macchioro ou de la vie privée du futur anthropologue, il vaut mieux s'interroger sur l'importance constitutive de cette langue évangélique que la relation épistolaire fait apparaître - au sein des divers cercles de parenté - comme un idiome, certes, partagé mais dont les usages, à l'instar des plus modernes langues charismatiques, sont nettement différenciés.

Les questions de méthode qui se posent au biographe pour restituer, dans le vécu existentiel et les intérêts intellectuels de l'ethnologue à venir, la prégnance de ces complexes productions de signes destinés à s'entreinterpréter, sont celles-là même que pose, à tout ethnologue, la description de dispositifs, sociaux ou rituels, qui font agir des êtres fictionnels. Plutôt que de mettre à distance, sous forme d'objet de croyance ou d'incroyance, la langue prophétique, la grammaire providentielle et les énoncés divinatoires qui circulent entre les deux locuteurs, il s'agit d'en décrire les contextes, les règles d'énonciation et les procédures de démétaphorisation qui les rendent opérants. Mais une difficulté supplémentaire se présente ici : nous n'observons pas directement les consultations de l'Évangile et de Sora Carlotta, nous avons seulement accès à des traces fragmentaires, fixées par l'écriture dans le travail interprétatif qu'elles déclenchent et qui alimente la relation épistolaire. Par exemple, associées aux transcriptions des « réponses » de l'Évangile et de la parole oraculaire de Sora Carlotta, Macchioro fait circuler entre Bari, Naples et Trieste les lettres que lui adresse De Martino, pour résoudre le violent conflit qu'a suscité la découverte d'un secret conjugal. Or, c'est bien, là, l'objet de notre enquête: non pas l'étude de cas d'un savoir culturel exemplifié dans un groupe familial, mais sa mise en tension dans l'activité psychique, et pas seulement dans l'effort conceptuel, du futur ethnologue. Se donnent, alors, à voir les différentes façons d'éprouver les très réels effets de ces traitements symboliques de l'infortune, dans la mesure où l'attitude critique qui sert, dans la modernité occidentale, de méta-communication pour transmettre ce savoir culturel, vient ici occuper le premier plan de l'espace de pensée construit par la relation épistolaire. 
Ces formes singulières de résolution d'épisodes de malheur domestique plongent, en effet, De Martino dans une crise intellectuelle analogue à la confusion qui saisit l'ethnographe sur son terrain, lorsqu'il est pris dans les discours et les gestes de ses interlocuteurs. Alors absorbé par la lecture critique de Lévy-Bruhl et de Croce pour élaborer les principes d'une ethnologie historiciste, il ne trouve pas de mots satisfaisants pour qualifier cette «complémentarité » offerte par son interlocuteur et qu'il éprouve, littéralement, comme une conversion du regard. Disciple et prophète, philosophe et mystique, théoricien et praticien? Aucun de ces couples de termes, tour à tour empruntés pour qualifier une forme énigmatique de transmission de savoir, ne semble répondre, de manière satisfaisante, au sentiment d'une altérité existentielle à conquérir sur le plan conceptuel : ce n'est que dans le climat libertaire de l'après-guerre qu'elle pourra prendre le nom de "rencontre» ethnographique. Retenons, alors, que par son étonnante aptitude à vivre et à penser en plusieurs régimes logiques, Vittorio Macchioro a fait au futur ethnologue un don infiniment précieux : le premier "terrain" où, malgré lui et dans la confusion, celui-ci s'est trouvé "pris " par des urgences pratiques analogues à celles de tout consultant. Parce qu'il s'est laissé affecter, dirait Jeanne Favret-Saada, par les effets de tous les médiateurs convoqués par son beau-père pour assurer la cohésion de ce groupe familial élargi, il peut se donner un programme intellectuel inédit: prendre au sérieux les compétences d'une grande variété d'experts, génériquement qualifiés de magiciens, certes éloignées dans une distance primitiviste mais, et c'est là l'essentiel, personnellement éprouvées comme détachées et libérées du jugement d'illusion.

Mais ce n'est pas tout. L'exploration d'un autre domaine d'activité créatrice du très inventif Macchioro s'impose pour donner tout son sens, chez De Martino, à un genre d'écriture brièvement adopté à l'abri d'un commun pseudonyme et qui, de ce fait, a échappé à tous les commentateurs: le récit romanesque pour penser une question d'une redoutable actualité à la veille de la Seconde Guerre mondiale, le devenir de la religion dans la modernité juive. L'altérité évangélique croise, en effet, une autre diversité découverte par le biais d'une autobiographie fictionnelle, terminée à la veille du départ en Inde, où, sous le nom de David Reubeni - une figure de faux messie dans la mémoire culturelle juive ${ }^{25}$ - son

25. David Reubeni apparaît à Venise en 1523, se disant d'origine éthiopienne et racheté sur un marché d'esclaves. Formé dans les écoles de Jérusalem et de Safed, il déclare être issu de l'ancienne tribu de Ruben dont il serait le dernier témoin. Le pape Clément VII et le roi Jean III de Portugal le reçoivent comme un ambassadeur, tandis que des communautés incrédules le rejettent. Le retour au judaïsme de familles marranes après son passage relance les persécutions. Reubeni finit par être arrêté et emprisonné en 1532, sur l'ordre de Charles Quint. Il meurt à Badajoz six ans plus tard. 
beau-père emprunte le modèle biographique du prophète méconnu et persécuté pour redonner sens à une identité rejetée, à mesure que se fait jour le retour d'un antisémitisme menaçant. Ce récit touffu de plus de sept cents pages raconte comment David Reubeni, présenté comme le descendant d'une famille de rabbins de Prague du XVII ${ }^{\mathrm{e}}$ siècle et qui devrait, à ce titre, devenir le gardien de la tradition, décide au contraire de devenir italien, séduit qu'il est par les beautés de l'Italie de la Renaissance. Mais, après une série de conversions religieuses et de catastrophes, puni par Dieu pour s'être donné une patrie, il quitte l'Italie pour une destination inconnue ${ }^{26}$. En inscrivant dans la trame individuelle d'une trajectoire d'effacement de l'être juif l'histoire religieuse d'un peuple, les signes de son anéantissement et les ressources culturelles déployées pour survivre, David Reubeni apparaît comme une sorte d'individu "apocalyptique " qui conjoint la fin d'un monde et la renaissance d'un autre monde. Il s'agit là, pour De Martino, d'une lecture bouleversante - elle l'occupe durant l'été 1935 - qui le confronte à l'immense question des contradictions de la solution libérale de l'assimilation. Trois ans plus tard, le voici qui expérimente à son tour les virtualités de l'écriture fictionnelle pour proposer une étonnante analyse de l'ethos religieux qui animerait les activités financières des juifs ayant choisi d'être "modernes en tout». En somme, une transposition de l'analyse webérienne des racines protestantes de l'esprit du capitalisme, dans une composition à deux mains d'un roman - Il Gioco di Satana ("Le Jeu de Satan»), 1939 - qui prolonge le récit autobiographique de son beau-père, sous le pseudonyme commun de Benedetto Gioia. L'écriture romanesque explore, en outre, le rapport général que chacun des personnages entretient avec un monde disloqué d'où la divinité semble s'être retirée, pour faire entendre des voix discordantes quant aux possibles relations entre judaïsme et christianisme.

Ce livre participe à une réconciliation familiale au moment où, avec la promulgation des lois raciales de septembre 1938, le gouvernement italien vient de s'aligner sur la politique de persécution antisémite de l'Allemagne nazie, politique qui touche directement Macchioro : rayé des fonctionnaires de l'État, il est mis d'office à la retraite en janvier 1939 avant d'être interné, en juin 1940, dans un camp des Marches. En reprenant l'histoire des Reubeni, De Martino s'écarte de la lecture laïcisée de l'antisémitisme moderne en général et des mesures mussoliniennes en particulier, pour interroger, sur le mode fictionnel, les rapports du christianisme avec son Autre le plus proche, et il participe aussi, de

26. Demeurée inédite, cette autobiographie a pour titre: Adonaï Sevaot. Le tapuscrit est conservé dans le Fonds Macchioro, Université de Trieste. On pourra lire la traduction d'un long synopsis dans Charuty (2009: 176-181). 
manière inattendue, à cette diversité triestine dont la différence juive condense les deux âmes. Vittorio Macchioro ainsi que l'éditeur Macrì, de Bari, espéraient convaincre un producteur de l'intérêt d'une adaptation cinématographique de ce roman. Après la guerre, c'est un autre monde en mouvement, celui de la Lucanie, qui relancera la quête d'un cinéaste, et il faudra attendre l'épuisement du travail ethnographique dans le Sud pour que la fin des mondes fasse retour dans le questionnement de l'anthropologue. Mais on ne peut plus, désormais, exclure du parcours d'apprentissage démartinien les effets de connaissance produits par cette intimité prolongée avec une judéité culturelle, plus que religieuse.

Il y a vingt ans, James Clifford nous a révélé l'effervescence artistique qui a favorisé la rencontre, dans le Paris des années 1920-1930, des préoccupations des ethnologues avec celles de l'avant-garde surréaliste, également convaincus de la nécessité de "désapprendre" nos codes culturels. De la même façon, nous pouvons inscrire la gestation de l'œuvre démartinienne dans un plus ample mouvement néoréaliste qui déplace dans le champ de la créativité culturelle les questions sous-jacentes aux premiers programmes d'enquête: le fascisme peut-il être une Troisième religion, une religion civile? La compréhension des magies lointaines peut-elle libérer l'Occident de la catastrophe totalitaire? Reconnaitre cette affinité n'est pas céder à l'illusion d'une linéarité biographique, où tous les moments d'une vie seraient pertinents pour donner sens à une œuvre. C'est rétablir l'historicité intérieure, les enchaînements imprévisibles d'actes de pensée et d'existence noués à une étonnante variété de pratiques d'écriture, choisies ou subies, pour défaire et refaire la personne même du chercheur, aux prises avec la fragmentation de relations de savoir traversées par une tension encore irrésolue entre le trop proche et le trop lointain. Ainsi De Martino a-t-il commencé par éprouver lui-même, avant de faire éprouver à tous ceux qu'il a placés en position de "contemporains", l'efficacité d'une multiplicité de façons d'écrire - épistolaire, documentaire, fictionnelle, biographique et autobiographique - pour rétablir la sphère de l'individualité au sein de ce changement civilisationnel dont il entendait décrire - sur le mode d'une ethnologie réformée - les principales scansions historiques.

"Nous sommes tous un peu fatigués du néoréalisme », fait dire Moravia à l'un des personnages du Mépris. De fait, comme pour le cinéma et la littérature, ce moment de l'anthropologie italienne qui a culminé avec les attentes placées dans la réception de l'expédition en Lucanie sera, à la fois, éphémère et décisif: le reconnaître permet de mieux apercevoir la diversité 
des voix nationales de refondation disciplinaire dans l'après-guerre. On le sait, le développement de l'ethnologie française sous le gouvernement de Vichy fut favorisé tant par les concessions initiales de quelques administrateurs, que par l'engagement propagandiste de quelques chercheurs dans la diffusion de la mythologie pétainiste. Après la guerre, une longue série de conjurations tint lieu d'analyse de ces compromissions : écarter le terme "folklore», adopter des problématiques strictement marxistes dans les enquêtes collectives et, à l'intérieur du musée-laboratoire, affirmer la neutralité scientifique de techniques de description qui excluent les amateurs (Fabre 1997). La voie italienne proposée et incarnée par De Martino a fait des choix exactement opposés pour "brancher» la différence ethnographique sur la vie quotidienne : le refus de l'espace purifié du musée, le désintérêt pour les objets, la déprofessionnalisation du savoir, le lien inversé mais ô combien réaffirmé entre productions culturelles et valeurs politiques, lequel exige une actualisation de l'analyse marxiste pour écarter la tentation d'un nouveau millénarisme. Et si la poétique de l'immédiateté que cette voie a valorisée ne fut qu'un moment de transition pour reconstruire la discipline, de ce singulier climat de participation existentielle et de détachement analytique, l'anthropologie démartinienne gardera une marque indélébile.

École pratique des hautes études de Paris Laboratoire d'anthropologie et d'histoire de l'institution de la culture (LAHIC), Charenton-le-Pont giordana.charuty@laposte.net

MOTS CLÉS/KEYWORDS: Ernesto De Martino - néoréalisme/neorealism - anthropologie italienne/ italian anthropology - religion civile/civil religion - fascisme/fascism - magie/magic cinéma italien/cinema of Italy. 
Ackerman, Robert

1987 J. G. Frazer. His Life and Work.

Cambridge, Cambridge University Press.

Amselle, Jean-Loup

\& Emmanuelle Sibeud, eds

1998 Maurice Delafosse. Entre orientalisme et ethnographie: l'itinéraire d'un africaniste, 1870-1926. Paris, Maisonneuve \& Larose.

\section{Asor Rosa, Alberto}

2002 L'Alba di un mondo nuovo.

Torino, Einaudi.

Barbaro, Umberto, ed.

1950 Il Cinema e l'uomo moderno.

Milano, Sociali.

Bazin, André

2007 Qu'est-ce que le cinéma?

Paris, Le Cerf-Corlet.

Boarelli, Mauro

2007 La Fabbrica del passato. Autobiografie di militanti comunisti (1945-1956).

Milano, Feltrinelli.

Calvino, Italo

1967 [1947] Il Sentiero dei nidi di ragno.

Torino, Einaudi.

\section{Carpitella, Diego}

1952 «Una spedizione etnologica in Lucania (30 sett.-31 ottobre) ", Società 8 (4) : 737-739.

Casajus, Dominique

2007 Henri Duveyrier. Un Saint-Simonien au désert. Paris, Ibis Press.

Casetti, Francesco \& Luca Malavasi

2003 "La retorica del neorealismo ", in Callisto Cosulich, ed., Storia del cinema italiano, 7 : 1945-1948. Venezia, MarsilioEd. di Bianco \& Nero : 176-190.
Cassac, Michel, ed.

2004 Littérature et Cinéma néoréalistes.

Réalisme, réel et représentation.

Paris, L'Harmattan.

\section{Charuty, Giordana}

2000 "L'ethnologue et le citoyen ", Gradhiva 26 : 83-98.

2009 Ernesto De Martino.

Les vies antérieures d'un anthropologue.

Marseille, Parenthèse-MMSH.

Clemente, Pietro, Maria Luisa Meoni

\& Massimo Squillacciotti

1976 Il Dibattito sul folklore in Italia. Milano, Ed. di Cultura popolare.

Clifford, James

1987 [1982] Maurice Leenhardt.

Personne et mythe en Nouvelle-Calédonie.

Paris, Jean-Michel Place.

1996 [1988] Malaise dans la culture.

L'ethnographie, la littérature et l'art au $X X^{e}$ siècle. Paris, École nationale supérieure des Beaux-Arts.

\section{Cole, Douglas}

1983 " "The Value of a Person Lies

in His Herzenbildung" : Franz Boas' Baffin Island Letter Diary, 1883-1884 ", in George W. Stocking, eds, Observers Observed. Essays on Ethnographic Fieldwork, History of Anthropology.

Madison, University of Wisconsin Press : 13-52.

De Felice, Renzo

2000 Les Interprétations du fascisme.

Introd. de Emilio Gentile.

Paris, Éd. des Syrtes.

Deleuze, Gilles

1985 Cinéma, 2 : L’image-temps.

Paris, Éd. de Minuit. 
De Martino, Ernesto

1941 Naturalismo e storicismo nell'etnologia. Bari, G. Laterza \& figli. [2 ${ }^{\mathrm{e}}$ éd. avec une introd. de Stefano De Matteis :

Lecce, Argo, 1995.]

1947 "Inchiesta di "Quarto Stato" sul mezzogiorno : Terra di Bari» (en collab. avec Mario Potenza), Quarto Stato 25-26: 32-36.

1948a "Cultura e classe operaia ", Quarto Stato 3 (1) : 19-22.

$1948 b$ "Amore cristiano e collera operaia ", Avanti! 52 (294), 12 dic. : 3.

1949a "La cultura cattolica ", Avanti!

53 (35), 10 febbr. : 3.

1949b «Libertas ", Avanti! 53 (48),

25 febbr. : 3.

1949c «Intorno a una storia del mondo popolare subalterno ", Società 5 : 411-435.

1950 «Note lucane", Società $6:$ 650-667.

1951a «Il Folklore: un invito ai lettori del "Calendario" ", Il Calendario del popolo $7: 989$.

$1951 b$ "Il folklore progressivo emiliano ", Emilia 3 : 251-254.

1952a «Nuie simme 'a mamma d' 'a bellezza ", Il Calendario del popolo 8 : 1061.

$1952 \mathrm{~b}$ "Gramsci e il folklore ", Il Calendario del popolo 8: 1109.

1952c « Il mondo popolare nel teatro di massa ", Emilia 1: 91-93.

1952d «Inchiesta sui costumi dei contadini lucani. 1 : Sonno, fame e morte sotto le stelle et 2 : Amore e morte nei canti dei braccianti lucani" (en collab. avec Benedetto Benedetti \& Arturo Zavattini), Vie Nuove 7 (30), 27 luglio : 12-13 et 7 (31), 3 agosto : 19.

1952e «Richiamo alla tradizione: teatro e spettacolo popolare ", Supplément à Vie Nuove 7 (42), 26 ott. : 28-29.

$1952 \mathrm{f}$ "Realismo e folklore nel cinema italiano ", Filmcritica 19: Testimonianze sul realismo: $183-185$.

$1952 \mathrm{~g}$ « La cultura nel mondo contadino meridionale ", Letture per tutti 4 (10) : 23-24.
1953 « Note di viaggio ", Nuovi Argomenti

1 (2) : 47-79. [Trad. franç. de Giordana Charuty, Daniel Fabre \& Carlo Severi in Gradhiva, 2000, 26 : 53-67.]

1966 [1961] La Terre du remords. Paris, Gallimard, 1966. [Rééd. : Paris, Institut Sanofi-Synthélabo, 1999 ("Les Empêcheurs de penser en rond ").]

1999 [1948] Le Monde magique.

Paris, Institut Sanofi-Synthélabo

(«Les Empêcheurs de penser en rond»).

2005 [1977] La Fine del mondo. Introd. de Clara Gallini \& Marcello Massenzio.

Torino, Einaudi.

\section{Di Donato, Riccardo}

1999 I Greci selvaggi. Antropologia storica di Ernesto de Martino. Roma, Manifestolibri.

Di Donato, Riccardo, ed.

1993 La Contraddizione felice? Ernesto De Martino e gli altri. Pisa, ETS.

\section{Downie, R. Angus}

1940 James George Frazer. The Portrait of a Scholar. London, Watts \& Co.

Fabre, Daniel

1997 "L'ethnologie française à la croisée des engagements, 1940-1945", in Jean-Yves Boursier, ed., Résistants et Résistance. Paris, L'Harmattan ("Chemins de la mémoire ») : 319-400.

2005 « "La Fine del mondo", une anthropologie de l'histoire ", in Marcello Massenzio \& Andrea Alessandri, eds, De Martino. Occidente e alterità.

Roma, Biblink ( Annali del dipartimento di storia» 1) : 57-76.

2007 «Le savoir des différences : histoire et science des mœurs en Europe

(XVIII ${ }^{\mathrm{e}}$-XXe siècles) ", in Karine Schemla \& Mireille Delbraccio, eds, Action concertée "Histoire des savoirs" 2003-2007. Recueil de synthèses. Paris, CNRS Éd.-Ministère de l'Enseignement supérieur et de la recherche : 65-68. 
Faeta, Francesco

1997 «Il sonno sotto le stelle : Arturo

Zavattini e le prime fotografie etnografiche demartiniane in Lucania ", Ossimori 8 : 57-67.

2003 Arturo Zavattini. Fotografo in

Lucania. Milano, Federico Motta.

Fardon, Richard

1999 Mary Douglas. An Intellectual

Biography. London-New York, Routledge.

Favret-Saada, Jeanne

1990 "Être affecté», Gradhiva 8 : 3-9.

Fournier, Marcel

1994 Marcel Mauss. Paris, Fayard.

2007 Émile Durkheim (1858-1917).

Paris, Fayard.

Gallini, Clara \& Francesco Faeta, eds

1999 I Viaggi nel Sud di Ernesto

De Martino. Torino, Bollati Boringhieri.

\section{Ginzburg, Natalia}

1963 Lessico famigliare. Torino, Einaudi.

\section{Gioia, Benedetto}

1939 Il Gioco di Satana. Bari, Macrì.

Gualtieri, Elena, ed.

2005 Paul Strand - Cesare Zavattini.

Lettere e immagini. Bologna, Bora.

Hinsley, Curtis

1983 «Ethnographic Charisma and Scientific Routine: Cushing and Fewkes in the American Southwest, 1879-1893 ", in George W. Stocking, ed., Observers Observed. Essays on Ethnographic Fieldwork. Madison, University of Wisconsin Press : 53-69.

Laurière, Christine

2008 Paul Rivet. Le savant et le politique. Paris, Muséum national d'histoire naturelle.

Leonesi, Luciano

1989 Il Romanzo del Teatro di Massa.

Bologna, Cappelli.
Levi, Carlo

1947 Cristo si è fermato a Eboli. Torino, Einaudi.

\section{Lukes, Steven}

1973 Emile Durkheim, His Life and Work. A Historical and Critical Study. Stanford, Stanford University Press.

Miccichè, Lino ed.

1975 Il Neorealismo cinematografico italiano. Venezia, Marsilio.

Mirizzi, Ferdinando, ed.

2009 Da vicino e da lontano.

Fotografi e fotografia in Lucania.

Milano, Franco Angeli.

Riley, A. Tristan

2001 «Ethnologie d'un anthropologue: à propos de la correspondance de Robert Hertz ", Gradhiva 30-31 : 123-134.

\section{Sasso, Gennaro}

2001 Ernesto De Martino fra religione e filosofia. Napoli, Bibliopolis.

Severino, Valerio

2002 «Ernesto De Martino nel circolo crociano di Villa Laterza, 1937-1942: contributo a una contestualizzazione politica de "Il mondo magico" ", La Cultura 40 (1) : 89-106.

\section{Stocking, George W. Jr.}

1983 "The Ethnographer's Magic: Fieldwork in British Anthropology from Tylor to Malinowski ", in George W. Stocking, ed., Observers Observed. Essays on Ethnographic Fieldwork. Madison, University of Wisconsin Press : 70-120.

\section{Szakolczai, Arpád}

1995 Durkheim, Weber and Parsons and the Founding Experiences of Sociology. San Domenico di Fiesole, European University Institute. 
Tinazzi, Giorgio \& Marina Zancan, eds 1990 [1983] Cinema e letteratura del neorealismo. Venezia, Marsilio.

Visconti, Luchino

1951 La Terra trema. Sceneggiatura. Introd. Luigi Chiarini. Roma, Bianco e Nero.
Vittorini, Elio

1977 Gli anni del "Politecnico".

Lettere, 1945-1951. Torino, Einaudi.
Young, Michael W.

2004 Malinowski. Odyssey

of an Anthropologist, 1884-1920.

New Haven, Yale University Press.

\section{RÉSUMÉ/ABSTRACT}

Giordana Charuty, Le moment néoréaliste de l'anthropologie démartinienne. - Quelle définition du biographique faut-il adopter pour restituer la construction interstitielle du savoir anthropologique, à travers la trajectoire existentielle d'un savant? Suspendre toute définition normative de ce qui, depuis Malinowski, est désigné comme "expérience de terrain" permet de reconnaître dans les multiples activités de De Martino antérieures aux expéditions ethnologiques dans le Sud italien, jusqu'ici écartées de l'histoire de la discipline ou qualifiées d'" anthropologie de cabinet", autant d'expériences de vie et de pensée qui prennent en charge les conditions de possibilité d'une refondation disciplinaire en étroite relation, au lendemain de la Seconde Guerre mondiale, avec le mouvement politico-culturel du néoréalisme.
Giordana Charuty, The Neorealistic Moment in De Martino's Anthropology. - What definition of biographies has to be adopted to restore the "interstitial construction " of anthropological knowledge through a scholar's existential trajectory? By suspending any normative definition of what (since Malinowski) has been called "experience in the field", we can detect in De Martino's many activities prior to his ethnological expeditions to southern Italy - till now omitted from the history of the discipline or qualified as "armchair anthropology" experiences that shaped his life and thought. They constitute the conditions for the possibility of a refoundation of the discipline in close relation with the post-WW II political, cultural movement of neorealism. 